\title{
Mayonnaise Increases the Relative Bioavailability of Fat-Soluble (Non)-Nutrients from a Salad
}

\author{
Fernanda de Oliveira Martins ${ }^{1}$, , Robin van den Berg ${ }^{2}$, Anne-Roos Hoogenraad ${ }^{2}$, \\ Ewoud Schuring ${ }^{2}$, Carole Verhoeven ${ }^{2}$, Oscar Giese Laverdy Neto ${ }^{3}$, Raul Cavalcante Maranhao ${ }^{3}$, \\ Petra Verhoef ${ }^{2}$ \\ ${ }^{1}$ Unilever Research and Development, Unilever Brazil, Sao Paulo, Brazil \\ ${ }^{2}$ Unilever Research and Development, Unilever Netherland, Vlaardingen, the Netherlands \\ ${ }^{3}$ Heart Institute (InCor) of the Medical School Hospital, University of Sao Paulo, Sao Paulo, Brazil
}

\section{Email address:}

fernanda.o.martins@unilever.com (F.de O. Martins), robin-van-den.berg@unilever.com (R. van den Berg), anne-roos.hoogenraad@unilever.com (A.-R. Hoogenraad), ewoud.schuring@unilever.com (E. Schuring), carole.verhoeven@unilever.com (C. Verhoeven), oscargiese@yahoo.com.br (O. G. L. Neto), ramarans@usp.br (R. C. Maranhao), petra.verhoef@unilever.com (P. Verhoef)

\section{To cite this article:}

Fernanda de Oliveira Martins, Robin van den Berg, Anne-Roos Hoogenraad, Ewoud Schuring, Carole Verhoeven, Oscar Giese Laverdy Neto, Raul Cavalcante Maranhao, Petra Verhoef. Mayonnaise Increases the Relative Bioavailability of Fat-Soluble (Non)-Nutrients from a Salad. International Journal of Nutrition and Food Sciences. Vol. 4, No. 6, 2015, pp. 644-649. doi: 10.11648/j.ijnfs.20150406.17

\begin{abstract}
Vegetables are good sources of vitamins. For that reason the World Health Organisation recommends daily consumption of at least $200 \mathrm{~g}$ of vegetables. However, this requirement is met only partially worldwide. Increasing the bioavailability of fat-soluble (non)-nutrients could make intake of those sources more effective. Here, the hypothesis was tested whether this goal can be achieved by adding mayonnaise to a dressing for a salad. Nine healthy subjects $(28.7 \pm 4.7$ years old, BMI of $26.7 \pm 2.1 \mathrm{~kg} / \mathrm{m}^{2}$ ) consumed a salad either with a basic dressing (control) or a basic dressing with mayonnaise (test). After both interventions, $\alpha$-carotene, $\beta$-carotene, vitamin $\mathrm{K}_{1}$ and retinyl palmitate were measured in chylomicron-rich plasma fractions. The average relative bioavailability $(-95 \% \mathrm{CI},+95 \% \mathrm{CI})$ was $+80 \%(-3 \%, 232 \%)$ for $\alpha$-carotene $(\mathrm{P}=0.0591),+91 \%$ $(22 \%, 200 \%)$ for $\beta$-carotene $(\mathrm{P}=0.0118)$, and $+116 \%(5 \%, 344 \%)$ for vitamin $\mathrm{K}_{1}(\mathrm{P}=0.0392)$. Retinyl palmitate AUC's increased by $117 \%(39 \%, 242 \%)(\mathrm{P}=0.0039)$, hence bioconversion of pro-vitamin A to retinol doubled. In conclusion, addition of mayonnaise to a dressing for a salad increased the uptake of the (non)-nutrients analysed in this study. Therefore, adding mayonnaise may be an interesting dietary approach to improve the nutritive value of vegetables, which are frequently underconsumed.
\end{abstract}

Keywords: Relative Bioavailability, Fat-Soluble (Non)-Nutrients, Carotene, Retinyl Palmitate, Vitamin $\mathrm{K}_{1}$, Mayonnaise, Dressing

\section{Introduction}

The World Health Organization (WHO) recommends a daily intake of at least $200 \mathrm{~g}$ of vegetables next to $200 \mathrm{~g}$ of fruit [1]. This is advised because fruits and vegetables constitute a group of plants that can be good sources of (non)-nutrients such as vitamins, minerals, carotenoids and flavonoids and fiber [2, 3]. However, in the case of vegetables the intake remains well below recommended amounts in some countries $[4,5]$. Lock et $a l$. reported that the combined average daily intake of fruit and vegetables in Latin America ranged from 72 to $235 \mathrm{~g}$ in 2005 for both genders and all age groups [5]. Fruit and vegetable consumption has been associated with lower risk of developing cardiovascular disease, cancer and other diseases $[6,7,8]$.

Fat-soluble vitamins contained in edible vegetables play important roles in promoting overall health. Among them are carotenoids $\alpha$ - and $\beta$-carotene, which are converted into retinol, the active form of vitamin $\mathrm{A}$ that functions as hormone and as the visual pigment of the eye. Vitamin $\mathrm{K}_{1}$, another important fat-soluble vitamin, is essential in the blood coagulation and bone mineralization processes [9].

Raising the intake of vegetables requires lifestyle changes which are often difficult to achieve [10]. However, the bioavailability of the fat-soluble (non)-nutrients contained in 
the vegetables can be increased by improving their luminal micellar solubility for absorption by the intestinal wall [11, 12, 13]. This may be accomplished by adding fats to the vegetables. In this regard, Brown et al. observed that the absorption of fat-soluble (non)-nutrients from salads can be increased by the addition of an oil-based salad dressing [12] and Takeda et al. reported that the isolated ingestion of broccoli with mayonnaise resulted in the increase of the a. $o$. $\beta$-carotene serum levels [14]. The addition of eggs to a salad with a variety of raw vegetables increased the absorption of carotenoids, as shown by Kim et al. [15].

To our knowledge, the effects of adding mayonnaise upon the uptake of (non)-nutrients from a salad composed by assorted vegetables has not yet been investigated. This is an important dietary issue, considering the nutritive potential of vegetables, the widespread availability of these foodstuffs and the need to improve the fat-soluble vitamin nutritional status of the population. Thus, this study was aimed to evaluate the effects of the addition of mayonnaise to a dressing for a standard salad upon the relative bioavailability of fat-soluble (non)-nutrients contained in the salad constituent vegetables.

\section{Methods}

The study was conducted at the Heart Institute of the Medical School Hospital, University of São Paulo, São Paulo, Brazil in accordance with applicable laws and regulations and with the ethical principles that have their origin in the Declaration of Helsinki, Finland. The protocol and informed consent were approved by the ethical committee for analysis of research projects of the Hospital das Clínicas of Universidade de São Paulo (University of São Paulo), Brazil. Written informed consent was obtained from study participants. The study was registered at clinicaltrials.gov (NCT01752764).

\subsection{Study Design and Participant Subjects}

This was a single-dose, single-blinded, randomized, full cross-over human nutrition intervention study. Fourteen subjects of both genders aged 18-60 years, with a BMI between 18.5 and $30.0 \mathrm{~kg} / \mathrm{m}^{2}$ and residing in the city of São Paulo, Brazil, were recruited. Subjects were screened through a medical interview on the basis of being apparently healthy. They had normal arterial blood pressure and reportedly none were smokers, addicted to alcohol consumption or had systemic arterial hypertension or liver, renal, thyroid, metabolic, inflammatory or neoplastic diseases. None were pregnant or lactating or using lipid-lowering, over-thecounter drugs or vitamin supplements. They were finally selected after serum biochemistry exams showing normal values of haemoglobin, total, LDL and HDL cholesterol and fasting triglycerides.

\subsection{Standardized Meal and Test Meal}

A standardized meal was provided to homes of the participant subjects and consisted of foods poor in carotenoids and vitamin $\mathrm{K} 1$ to ensure insignificant interference with the products analysed in this study. The participant subjects were instructed to consume the standardized meal the day before the intervention and to keep a 12-h fasting before consuming the test meal.

The test meal consisted of a test salad with either a control dressing or a dressing with mayonnaise. The test salad was made from $40 \mathrm{~g}$ fresh Romaine lettuce, $85 \mathrm{~g}$ fresh Carmen tomato, $66 \mathrm{~g}$ shredded fresh Nantes carrots, $10 \mathrm{~g}$ fresh Walking Stick kale, and 35 g cooked New Zealand spinach. The control dressing contained $40 \mathrm{~g}$ full-fat yoghurt, $9 \mathrm{~g}$ mustard, $4 \mathrm{~g}$ honey and $4 \mathrm{~g}$ soy sauce. The dressing with mayonnaise contained, next to previously mentioned ingredients, $24 \mathrm{~g}$ mayonnaise (Table 1).

Table 1. Composition of the control dressing and of the dressing with mayonnaise to each salad portion.

\begin{tabular}{lllll}
\hline & \multicolumn{2}{l}{ Control Dressing } & \multicolumn{2}{l}{$\begin{array}{l}\text { Dressing with } \\
\text { mayonnaise }\end{array}$} \\
\hline Ingredient & $\begin{array}{l}\text { Total } \\
\text { amount (g) }\end{array}$ & $\begin{array}{l}\text { Fat Amount } \\
\text { (g) }\end{array}$ & $\begin{array}{l}\text { Total } \\
\text { amount (g) }\end{array}$ & $\begin{array}{l}\text { Fat Amount } \\
\text { (g) }\end{array}$ \\
\hline $\begin{array}{l}\text { Full-fat } \\
\text { Yogurt }^{\mathrm{a}}\end{array}$ & 40.0 & 1.6 & 40.0 & 1.6 \\
Mustard $^{\mathrm{b}}$ & 9.0 & - & 9.0 & - \\
Honey $^{\mathrm{c}}$ & 4.0 & - & 4.0 & - \\
Soy Sauce $^{\mathrm{d}}$ & 4.0 & - & 4.0 & - \\
Mayonnaise $^{\mathrm{e}}$ & 0 & - & 24.0 & 8.0 \\
\hline
\end{tabular}

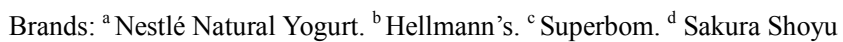
Light. ${ }^{e}$ Hellmann's, containing $32.5 \%$ fat (60 \% PUFA, $25 \%$ MUFA, $15 \%$ SFA, $0 \%$ trans fat).

The participant subjects received two test salads that had identical composition but that had the control dressing or the dressing with mayonnaise. The dressings with the test salads were ingested in a random order, each separated by a washout period of seven days. Both test meals were consumed together with maximum $250 \mathrm{~mL}$ water and with $12.5 \mathrm{~g}$ of white bread within half an hour.

Samples of $10 \mathrm{~mL}$ of whole blood were taken via an intravenous cannula at $0.25 \mathrm{~h}$ before and 2.0, 3.5, 5.0, 7.0 and $9.5 \mathrm{~h}$ after treatments were given. Immediately after blood collection the chylomicron fractions were isolated as described earlier [16] and stored at $-70{ }^{\circ} \mathrm{C}$ for later analysis.

\subsection{Analytical Procedures}

Samples of the test salad and both dressings were analysed at TNO Triskelion, Zeist, the Netherlands for $\alpha$-carotene, $\beta$ carotene, vitamin $\mathrm{K}_{1}$ and retinol, moisture and total fat, using established methods. In addition, chylomicron fractions samples were measured for $\alpha$-carotene, $\beta$-carotene, vitamin $K_{1}$ and retinyl palmitate at the Heart Institute of the Medical School Hospital using HPLC-MS-MS as described earlier [16].

\subsection{Relative Bioavailability and Statistical Analysis}

From the plasma concentration-time curve for $\alpha$-carotene, $\beta$-carotene, vitamin $\mathrm{K}_{1}$ and retinyl palmitate, AUC's were 
calculated using the trapezoidal rule. From this the relative bioavailability for $\alpha$-carotene, $\beta$-carotene and vitamin $\mathrm{K}_{1}$ were calculated for dressing with mayonnaise versus control dressing. Differences were evaluated using twosided analysis of covariance. Individual components using the baseline values per subject as a covariate, subjects as random factor, visit and their interaction as fixed factors. Significance was considered for $p$ values $<0.05$. Statistical analyses were performed using JMP Pro 11.0.0 (SAS Institute Inc., Cary, NC) and SAS software version 9.2 (SAS Institute, Cary, NC).

\section{Results}

\subsection{Study Subjects}

Fourteen subjects were screened and twelve (six males, six females) met the inclusion selection criteria. Three subjects received dressings, for both test meals, prepared with skimmed yoghurt instead of the prescribed full-fat yoghurt. Data from those subjects were discarded. The remaining nine subjects received correct test meals and completed the study.
All subjects finished the consumption of the meals within half an hour. The nine remaining subjects (four males, five females) were $28.7 \pm 4.7$ years old and had BMI of $26.7 \pm$ $2.1 \mathrm{~kg} / \mathrm{m}^{2}$.

\subsection{Amounts of (Non)-Nutrients in Test Meals}

Four representative samples of the test salads, two control dressings and two dressings with mayonnaise were taken for nutrient analysis. Data of the (non)-nutrients content of the test salad, of the control dressing and the dressings with mayonnaise are shown in Table 2.

Table 2. Amounts of (non)-nutrients in the test salad, control dressing and the dressing with mayonnaise.

\begin{tabular}{llll}
\hline $\begin{array}{l}\text { Nutrient } \\
\text { amount }\end{array}$ & $\begin{array}{l}\text { Salad* } \\
(\mathbf{2 3 6 g})\end{array}$ & $\begin{array}{l}\text { Control } \\
\text { dressing** }\end{array}$ & $\begin{array}{l}\text { Dressing with } \\
\text { mayonnaise** }\end{array}$ \\
\hline$\alpha$-Carotene $(\mu \mathrm{g})$ & 1793 & $<1$ & $<2$ \\
$\beta$-Carotene $(\mu \mathrm{g})$ & 7269 & 10 & 14 \\
Retinol $(\mu \mathrm{g})$ & 12 & $<1$ & 24 \\
Vitamin $\mathrm{K}_{1}(\mu \mathrm{g})$ & 157 & $<1$ & 17 \\
\hline
\end{tabular}

*Values are means $(\mathrm{n}=4) . * *$ Values are means $(\mathrm{n}=2)$
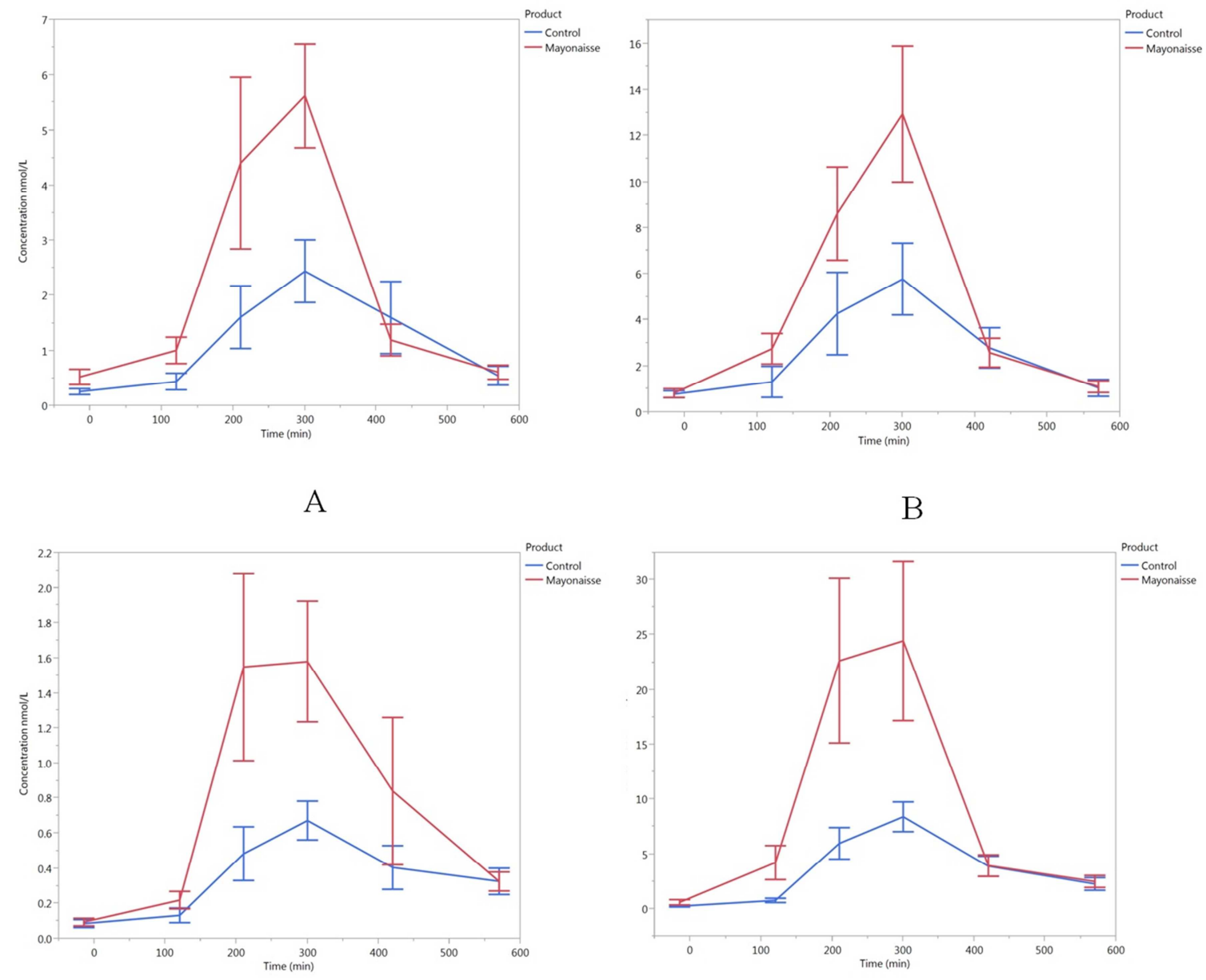

$\mathrm{B}$

$\mathrm{C}$

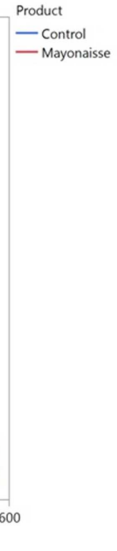

A

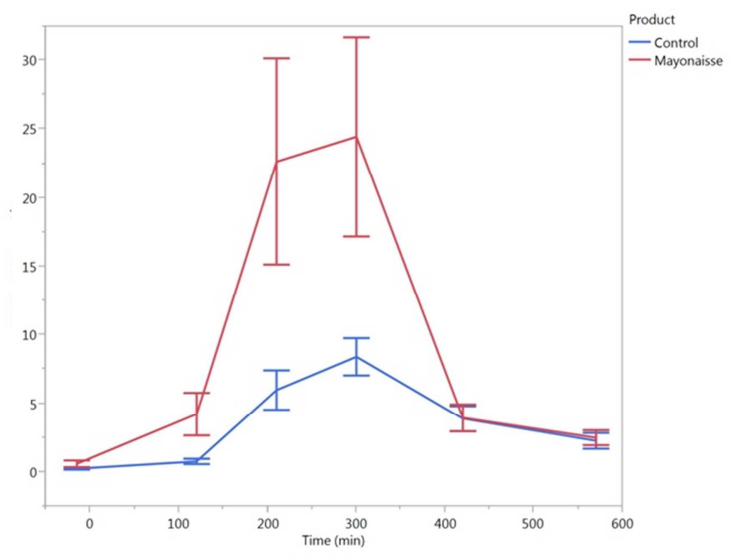

D

Figure 1. Concentrations of $\alpha$-carotene (A), $\beta$-carotene (B), retinyl palmitate $(C)$, vitamin $K_{1}(D)$ in chylomicron-rich fractions of plasma after a salad with the control dressing (blue) or the dressing with mayonnaise (red) was given. Points and bars represent means \pm Standard Error. 


\subsection{Plasma Concentration-Time Curves}

In Figure 1, the plasma concentration curves of $\alpha$-carotene (A), $\beta$-carotene (B), retinyl palmitate (C) and vitamin $\mathrm{K}_{1}$ (D) obtained at baseline $(\mathrm{t}=0 \mathrm{~h})$ and after the consumption of the salad with the control dressing (blue) or with the dressing with mayonnaise (red) from the nine participant subjects. Points and bars represent means \pm S.E.

\subsection{Relative Bioavailabilities}

The average percentual difference in AUCs for salads eaten with dressing with mayonnaise versus control dressing (i.e. relative bioavailability) for the (non)-nutrients are presented in Table 3. AUC's retinyl palmitate increased by $117 \%$ (lower $95 \% \mathrm{CI}=39 \%$, upper $95 \%$ CI $=242 \%$, $\mathrm{P}=0.0039$ ).

Table 3. Percentual differences in relative bioavailabilities of selected (non)nutrients for salad eaten with dressing with mayonnaise versus control dressing.

\begin{tabular}{lll}
\hline (non)-nutrient & $\begin{array}{l}\text { Difference in relative } \\
\text { bioavailability }(\%)\end{array}$ & P-value** \\
\hline$\alpha$-Carotene & $+80(-3,+232)$ & 0.0591 \\
$\beta$-Carotene & $+91(+22,+200)$ & 0.0118 \\
Vitamin K1 & $+116(+5,+344)$ & 0.0392 \\
\hline
\end{tabular}

*Averages of $\mathrm{n}=9$ are given, with upper $95 \%$ CI's, lower $95 \%$ CI's.

$* *$ Significance was declared for $\mathrm{P}$ values $<0.05$.

\section{Discussion}

Mayonnaise is a worldwide consumed tasty product, basically consisting of an oil-in-water emulsion made with vegetable oils, with addition of eggs, lemon juice or vinegar and salt. In this study, the ability of a commercial regular mayonnaise to facilitate the absorption of (non)-nutrients from a salad was investigated for the first time.

The main finding of the current study was that the addition of a mayonnaise to a dressing increased by $80 \%$ the absorption of $\alpha$-carotene, by $91 \%$ that of $\beta$-carotene, and by $116 \%$ that of vitamin $\mathrm{K}_{1}$ from a test salad. In addition, it raised bioconversion of carotenoids to retinyl palmitate by $117 \%$. The marked increase in the absorption of those selected fat-soluble (non)-nutrients confirms the value of this product to improve the nutritious value of edible vegetables.

In several countries, the intake of vegetables by the general population is often below the WHO recommendations [5] due to enrooted dietary habits. Since it is difficult to change dietary behaviour [10] a tentative strategy consists in increasing the intestinal absorption of (non)-nutrients contained in the vegetables to at least partially compensate for the lower intake. In this regard, the addition of fat to vegetables has been proven to pronouncedly increase the intestinal absorption of (non)-nutrients. In the intestinal lumen, long chain fatty acids form micelles including the (non)-nutrients that facilitate the crossing of the non-stirred water layer and the uptake by the enterocytes. The absorbed (non)-nutrients will be incorporated into nascent chylomicrons, which are secreted in the lymph and subsequently in the bloodstream, where they undergo lipolysis by lipoprotein lipase on the endothelial surface of capillaries. The resulting chylomicron remnants containing the (non)-nutrients are finally taken-up by the liver.

The role of fats facilitating the absorption of the fat soluble nutrients by the gut was previously documented $[13,17]$ and appears as a dose-dependent variable [12]. The effects of long-chain triglycerides (LCT), which are the main fat constituents of the mayonnaise consumed by the participants of our study, as compared to medium-chain triglycerides (MCT), was investigated by Borel et al. [17]. In that study, subjects were given a test meal containing $120 \mathrm{mg} \beta$-carotene incorporated into $40 \mathrm{~g}$ LCT meal or $40 \mathrm{~g}$ MCT meal. Chylomicron $\beta$-carotene and retinyl palmitate responses were dramatically diminished when the subjects ingested $\beta$ carotene with MCTs rather than LCTs.

It is worthwhile to point-out that retinyl palmitate has classically been used as marker of chylomicrons and their remnants in the circulation, since this compound does not dissociate from chylomicron particles until their final uptake by the liver [18]. As shown in Fig. 1C, after the ingestion of the test salad, the retinyl palmitate peaks of salads with or without dressing were at the same range, from 3-5 hours. Similarly, the rapid decay of the retinyl palmitate curves that occurred after the 5 hour point was equal. This indicates that although the amount chylomicrons in the circulation was greater under consumption of the salad with dressing, saturation of the hepatic mechanisms that remove remnants from the plasma did not occur at this level of fat ingestion. This issue may be potentially of interest since delayed removal of chylomicron remnants has been associated to the presence of atherosclerosis $[18,19]$ and to complications of coronary artery disease [20].

In the current study, a remarkable improvement of the availability of the fat-soluble (non)-nutrients was achieved with the consumption of a product with rather nutritionally favourable features. In this respect, the composition of the mayonnaise used in the current study had low \% total fat $(32.5 \%)$ whereas the fatty acid profile totally conforms to the recommended by WHO aiming to promote the prevention of cardiovascular disease [21], with low saturated fatty acid (SFA) and high poly-unsaturated fatty acid (PUFA) and mono-unsaturated fatty acid (MUFA) contents and with virtual absence of trans fatty acids. Also, the caloric intake relative to the amount of added mayonnaise was rigorously within the requirements of healthy and weight management diets. This can be assumed since the added mayonnaise delivers $80 \mathrm{kcal}$, which stands for only $4 \%$ of the total caloric intake in a base of daily $2000 \mathrm{kcal}$. For comparative ends, one tablespoon of vegetable oil delivers at least twice energy than one tablespoon of this mayonnaise.

In our experiments, the volume of the dressing with mayonnaise was larger than that of the control dressing due to the added $24 \mathrm{~g}$ mayonnaise. This is equivalent to two 
tablespoons of mayonnaise, which can be considered a reasonable volume for the amount of salad offered to the participants. The control dressing was not corrected for volume and calorie content corresponding to the added mayonnaise because this factor would be unlikely to influence the results. Another important issue for the interpretation of the results was that the added mayonnaise had only trace amounts of vitamin $\mathrm{K}_{1}$ and carotenoids.

\section{Conclusions}

Our results showed that by adding a mayonnaise to a salad facilitates the uptake of $\alpha$-carotene, $\beta$-carotene and vitamin $\mathrm{K}_{1}$ and thereby may improve the nutritional status of populations with vegetable consumption below the recommended figures. Furthermore, mayonnaise addition can reach higher fat soluble (non)-nutrient bioavailability with a fat profile and low caloric density that favours a healthy and weight management dietary pattern.

\section{List of Abbreviations}
WHO: World Health Organization
BMI: Body mass index;
LDL: Low density lipoprotein;
HDL: High density lipoprotein;
PUFA: Polyunsaturated fatty acid;
MUFA: monounsaturated fatty acid;
SFA: saturated fatty acid;
AUC: Areas under the response curve;
LCT: long-chain triglycerides;
MCT: medium-chain triglycerides.

\section{Acknowledgments}

We thank Renata Do Val for coordination of the study and Wanderlei Santos Monteiro Gomes for technical assistance; both from Heart Institute (InCor).

\section{References}

[1] World Health Organization (WHO), Diet, nutrition and the prevention of chronic diseases. 2003.

[2] H. Boeing, A. Bechthold, A. Bub, S. Ellinger, D. Haller, A. Kroke, et al., Critical review: vegetables and fruit in the prevention of chronic diseases. Eur J Nutr 2012; 51: 637-63.

[3] Nutrient Data Laboratory USDA-ARS, USDA National Nutrient Database for Standard Reference. 2015.

[4] Ministério da Saúde, Secretaria de Atenção à Saúde, Departamento de Atenção Básica. Coordenação Geral da Política de Alimentação e Nutrição. Guia alimentar para a população brasileira. Brasília, Brasil. 2006 (In Portuguese).

[5] K. Lock, J. Pomerleau, L. Causer, D. R. Altmann, M. McKee, The global burden of disease attributable to low consumption of fruit and vegetables: implications for the global strategy on diet. Bull World Health Organ 2005; 83: 100-8.
[6] F. J. He, C. A. Nowson, M. Lucas, G. A. MacGregor, Increased consumption of fruit and vegetables is related to a reduced risk of coronary heart disease: meta-analysis of cohort studies. J Hum Hypertens 2007; 21: 717-28.

[7] S. C. Larsson, J. Virtamo, A. Wolk, Total and specific fruit and vegetable consumption and risk of stroke: a prospective study. Atherosclerosis 2013; 227: 147-52.

[8] World Health Organization (WHO), Global Strategy on Diet, Physical Activity and Health. Promoting fruit and vegetable consumption around the world. 2014.

[9] S. S. Gropper, J. L. Smith, J. L. Groff, Advanced nutrition and human metabolism. Check edition ed. 2015.

[10] L. Gerchow, B. F. Tagliaferro, A. F. Squires, J. F. Nicholson, S. M. Savarimuthu, D. Gutnick, et al., Latina food patterns in the United States: a qualitative metasynthesis. Nursing Research 2014; 63: 182-193.

[11] A. J. Roodenburg, R. Leenen, K. H. van het Hof, J. A. Weststrate, L. B. Tijburg, Amount of fat in the diet affects bioavailability of lutein esters but not of alpha-carotene, betacarotene, and vitamin E in humans. Am J Clin Nutr 2000; 71: 1187-93.

[12] M. J. Brown, M. G. Ferruzzi, M. L. Nguyen, D. A. Cooper, A. L. Eldridge, S. J. Schwartz, et al., Carotenoid bioavailability is higher from salads ingested with full-fat than with fat-reduced salad dressings as measured with electrochemical detection. Am J Clin Nutr 2004; 80: 396-403.

[13] S. R. Goltz, W. W. Campbell, C. Chitchumroonchokchai, M. L. Failla, M. G. Ferruzzi, Meal triacylglycerol profile modulates postprandial absorption of carotenoids in humans. Mol Nutr Food Res 2012; 56: 866-77.

[14] S. Takeda, Y. Masuda, M. Usuda, R. Marushima, T. Ueji, M. Hasegawa, C. Maruyama, Effects of mayonnaise on postprandial serum lutein/zeaxanthin and beta-carotene concentrations in humans. J Nutr Sci Vitaminol (Tokyo) 2009 Dec; 55: 479-85.

[15] J. E. Kim, S. L. Gordon, M. G. Ferruzzi, W. W. Campbell, Effects of egg consumption on carotenoid absorption from co-consumed, raw vegetables. Am J Clin Nutr 2015; 102(1): 75-83.

[16] R. E. Kopec, R. M. Schweiggert, K. M. Riedl, R. Carle, S. J. Schwartz, Comparison of high-performance liquid chromatography/tandem mass spectrometry and highperformance liquid chromatography/photo-diode array detection for the quantitation of carotenoids, retinyl esters, alpha-tocopherol and phylloquinone in chylomicron-rich fractions of human plasma. Rapid Commun Mass Spectrom 2013; 27: 1393-402.

[17] P. Borel, V. Tyssandier, N. Mekki, P. Grolier, Y. Rochette, M. C. Alexandre-Gouabau, D. Lairon, V. Azais-Braesco, Chylomicron beta-carotene and retinyl palmitate responses are dramatically diminished when men ingest beta-carotene with medium-chain rather than long-chain triglycerides. J Nutr 1998; 128: 1361-7.

[18] M. S. Weintraub, I. Grosskopf, T. Rassin, H. Miller, G. Charach, H. H. Rotmensch, M. Liron, A. Rubinstein, A. Iaina, Clearance of chylomicron remnants in normolipidaemic patients with coronary artery disease: case control study over three years. BMJ. 1996; 12: 935-9. 
[19] R. C. Maranhão, M. C. Feres, M. T. Martins, C. H. Mesquita, O. Toffoletto, C. G. Vinagre, S. D. Gianinni, F. Pileggi, Plasma kinetics of a chylomicron-like emulsion in patients with coronary artery disease. Atherosclerosis. 1996; $126(1): 15-25$.

[20] A. C. Sposito, P. A. Lemos, R. D. Santos, W. Hueb, C. G. Vinagre, E. Quintella, O. Carneiro, M. J. Chapman, J. A. Ramires, R. C. Maranhão, Impaired intravascular triglyceride lipolysis constitutes a marker of clinical outcome in patients with stable angina undergoing secondary prevention treatment: a long-term follow-up study. J Am Coll Cardiol. 2004; 16; 43(12): 2225-32.

[21] Food and Agriculture Organization of the United Nations (FAO), World Health Organization (WHO), Interim Summary of Conclusions and Dietary Recommendations on Total Fat \& Fatty Acids. 2008. 